Trauma Berufskrankh 2008 • 10[Suppl 1]:85-90 DOI 10.1007/s10039-007-1305-5

Online publiziert: 2. Dezember 2007

๑) Springer Medizin Verlag 2007

\author{
A. Ekkernkamp ${ }^{1}$ - E. Haider ${ }^{1,2} \cdot$ E. Froese $^{2} \cdot$ D. Stengel ${ }^{1}$ \\ ${ }^{1}$ Zentrum für Klinische Forschung, Unfallkrankenhaus, Berlin \\ ${ }^{2}$ Verwaltungs-Berufsgenossenschaft, Hamburg
}

\title{
Qualität und Nutzen der Heilverfahren
}

stecken noch weitgehend in der Etablierungs- und Modellphase; ihr Einfluss auf die medizinische Versorgung und das Gesundheitsbild der Bevölkerung bleibt zu prüfen. Gleiches gilt für die langfristigen Folgen der pauschalierten Vergütung unter DRG-Bedingungen und der Errichtung des Instituts für Qualität und Wirtschaftlichkeit im Gesundheitswesen (IQWIG).

In den letzten Jahren haben sich zunehmend Schnittmengen aus Strömungen gebildet, die bereits direkten Einfluss auf die Entwicklung des öffentlichen Gesundheitswesens nehmen oder noch nehmen werden. Zu nennen sind, allen voran, der Qualitätsgedanke und verschiedene Methoden der Qualitätssicherung. Unmittelbare Grenzgebiete sind unter anderem die evidenzbasierte Medizin und die Gesundheitstechnologiebewertung.

\section{Der Qualitätsgedanke}

Qualitativ hochwertige Medizin ist keine Folge administrativer Vorgaben, sondern ein historisch gewachsener, zentraler Bestandteil des ärztlichen Selbstverständnisses. Ärzte werden Qualität häufig anders definieren als die Anspruchnehmer und Kostenträger von Gesundheitsleistungen.

Nach DIN 55350 und ISO 8402 ist Qualität „die Gesamtheit der Merkmale und Merkmalswerte eines Produktes oder einer Dienstleistung, festgelegte und vorausgesetzte Erfordernisse entsprechend ihrer Eignung zu erfüllen."

Seit Januar 1993 ist Qualitätssicherung als unverzichtbarer Bestandteil der ärztlichen Versorgung im SGB V rechtsverbindlich vorgeschrieben. Die gesetz- lich geforderten Maßnahmen sind „auf die Qualität der Behandlung, der Versorgungsabläufe und der Behandlungsergebnisse zu erstrecken. Sie sind so zu gestalten, dass vergleichende Prüfungen ermöglicht werden."

Einigkeit besteht national und international über die drei Dimensionen von Qualität:

- Strukturqualität,

- Prozessqualität und

- Ergebnisqualität.

Die kritische Masse wird durch die Ergebnisqualität bestimmt.

So wird z. B. eine ausschließliche Verkürzung von Prozessabläufen durch Großgerätetechnologie, die keinen positiven Einfluss auf Komplikations- und Heilungsraten nimmt, kaum als relevanter Qualitätsindikator herhalten können.

Biologische Systeme folgen allen möglichen Gesetzmäßigkeiten, ob linear, komplex oder chaotisch - sicher jedoch nicht den Industrienormen. Dennoch sind eine Simplifizierung und eine einheitliche Terminologie wertvoll, um überhaupt zu einer praktischen Umsetzung des Qualitätsgedankens in der Medizin zu kommen.

Zahlreiche Versuche, die Qualität der medizinischen Versorgung zu umreißen (http://www.medizinfo.de/quality/html/), möchten wir um den folgenden Minimalkonsens ergänzen:

Qualität im Gesundheitswesen ist die Summe aller messbaren, reproduzierbaren, erwünschten Einflüsse auf die Gesundheit einzelner Personen oder einer Bevölkerung als Folge gezielt eingesetzter, allgemein akzeptierter und nachweis- 
Tab. 1 Verschiedene Ebenen der Gesundheitstechnologiebewertung. Anwendung und Interpretation müssen hierarchisch erfolgen:

Eine Methode muss zuerst ihre Wirksamkeit unter Beweis stellen, bevor sie zur Prüfung auf den nächsten Ebenen zugelassen wird

\begin{tabular}{|c|c|c|c|}
\hline Ebene & $\begin{array}{l}\text { Wirksamkeit } \\
\text { („,efficacy“) }\end{array}$ & $\begin{array}{l}\text { Wirkung } \\
\text { (,effectiveness") }\end{array}$ & $\begin{array}{l}\text { Effizienz } \\
\text { („efficiency“) }\end{array}$ \\
\hline Typisches Studiendesign & Klinisches Experiment (RCT) & $\begin{array}{l}\text { Administrative Daten, Register, Präferenz- } \\
\text { studien }\end{array}$ & Gesundheitsökonomische Modelle \\
\hline $\begin{array}{l}\text { Typisches therapeutisches } \\
\text { Problem }\end{array}$ & Ist A besser als Plazebo? & Ist $A$ besser als $B$ ? & $\begin{array}{l}\text { Führt A zu einer höheren Ergebnisqualität } \\
\text { auf dem Niveau des Individuums oder der } \\
\text { Gesellschaft? }\end{array}$ \\
\hline $\begin{array}{l}\text { Typisches diagnostisches } \\
\text { Problem }\end{array}$ & $\begin{array}{l}\text { Misst der Test, was er zu } \\
\text { messen vorgibt? (Genauigkeit, } \\
\text { Sensitivität/Spezifität) }\end{array}$ & $\begin{array}{l}\text { Hat das Testergebnis eine } \\
\text { therapeutische Konsequenz? }\end{array}$ & $\begin{array}{l}\text { Verbessert die aus dem Testergebnis abge- } \\
\text { leitete Konsequenz die Ergebnisqualität? }\end{array}$ \\
\hline
\end{tabular}

lich sicherer Diagnose- und Behandlungsmethoden.

Aus ärztlicher Perspektive sollte sich Qualität immer als Verlängerung oder Verbesserung des Lebens ausdrücken lassen. Offensichtlich ergeben sich hierbei starke Überlappungen mit der Nutzenbewertung medizinischer Maßnahmen und der Versorgungsforschung.

Die Autoren sehen trotz einer sicher wünschenswerten Begriffstrennung keinen Konflikt zwischen Qualität und Nutzen, insbesondere dann, wenn die Ergebnisqualität in den Vordergrund gestellt wird. Eine nützliche Maßnahme sollte auch geeignet sein, die Qualität der Versorgung zu verbessern. Gleichzeitig sollten in einem qualitativ hochwertigen Gesundheitssystem auch nur Verfahren mit wissenschaftlich gesicherter Wirksamkeit zur Anwendung kommen - ansonsten würde Qualität unbezahlbar.

\section{Qualität und Nutzen}

Wenn Gesundheit als Gut und ihre Erhaltung und Wiederherstellung als (Dienst-) Leistung betrachtet werden, muss auch ihre Bilanzierung einfachen ökonomischen Prinzipien folgen. Eine Methode darf nur dann akzeptiert und solidarisch finanziert werden, wenn der zu erwartende Nutzen in einem angemessenen Verhältnis zu den zu erwartenden Kosten steht. Grundsätzlich sind sechs Szenarien im medizinischen Alltag denkbar:

1. Eine neue Methode ist schlechter und teurer als die etablierte.

2. Eine neue Methode ist gleichwertig mit der etablierten, aber teurer.

3. Eine neue Methode ist besser und billiger als die etablierte.
4. Eine neue Methode ist schlechter, aber billiger als die etablierte.

5. Eine neue Methode ist gleichwertig mit der etablierten, aber billiger.

6. Eine neue Methode ist besser, aber teurer als die etablierte.

Ein funktionierendes Gesundheitssystem muss Maßnahmen treffen, um das erste Szenario in jedem Fall zu vermeiden. Möglicherweise erlaubt erst eine hohe Struktur- und Prozessqualität, unwirksame, kostenintensive Systemkomponenten $\mathrm{zu}$ erkennen und rasch zu eliminieren.

Das zweite Szenario ist problematisch. So führt z. B. der starke Konkurrenzdruck zwischen den Implantatherstellern (wie in jedem anderen Industriezweig auch) da$\mathrm{zu}$, dass in regelmäßigen Abständen Osteosynthesematerial vorgestellt und vermarktet wird, welches sich lediglich in unbedeutenden Details von der zugelassenen und etablierten Technologie unterscheidet. Dennoch entstehen Entwicklungskosten, die auf den Preis der Implantate umgeschlagen werden.

Es erfordert neue, interdisziplinäre Methoden, um den Beitrag dieser Komponenten zur Versorgungsqualität messen und bewerten zu können.

Das dritte Szenario beschreibt den Idealfall. Es fragt sich jedoch, wie wahrscheinlich es ist, dass angesichts des erreichten medizinischen Fortschritts noch einmal eine bessere und gleichzeitig billigere Therapie entwickelt werden kann.

Die Grenzen zwischen Szenario 4 und 5 sind fließend. Insbesondere in der pharmakologischen Forschung ist es üblich, vor der Zulassung von Generika sog. Nichtunterlegenheits- oder auch Äquivalenzstudien durchzuführen. Da es keine absolut identisch wirksamen Interventionen gibt, gelten Unterschiede, die ein vorher festgelegtes Intervall nicht überschreiten, als vernachlässigbar und die getesteten Substanzen als gleichwertig. So könnte z. B. ein Medikament B zu einer Heilungsrate von $75 \%$ führen, während das bisher eingesetzte Medikament A regelhaft eine Heilungsrate von $80 \%$ erreichte. Liegt die Differenz von $5 \%$ innerhalb der Toleranzgrenzen, gelten $\mathrm{A}$ und $\mathrm{B}$ als gleich, obwohl $B$ in der Prüfung schlechter abgeschnitten hat. Ist B erheblich billiger als A, erfordert es einen tragfähigen Konsens zwischen Politik, Kostenträgern, medizinischen Fachorganisationen und Betroffenen, um A durch B zu ersetzen.

Das letztgenannte Szenario ist das derzeit am häufigsten vorkommende, leider aber auch das problematischste. Hierbei muss die Chronologie streng eingehalten werden - „besser, aber teurer“ kommt immer vor „teurer, aber besser.“

Die Anzahl von Entwicklungen in der Biomedizin folgt seit Jahrzehnten einer Exponentialfunktion. Die Flut wissenschaftlicher Veröffentlichungen ist auch für Experten nicht mehr zu bewältigen allein die Datenbank Medline enthält aktuell mehr als 17 Mio. Zitate, Tendenz steigend. Echte Innovationen sind demgegenüber eine Rarität. Die Balance zwischen euphorisch vorgeschlagenen und vermarkteten Neuerungen und der raschen Ernüchterung durch marginale, keine, oder gar schädliche Effekte im Vergleich zu etablierten Methoden ist bestenfalls instabil. Nach derzeitigen Schätzungen gelangen allenfalls ein Viertel aller Neuentwicklungen in die Phase der klinischen Prüfung, lediglich ein Zehntel auch in den klinischen Einsatz [1]. 


\section{Abnehmender Grenznutzen}

Die Innovationsspirale ist nicht beliebig schnell drehbar - sie wird durch den sog. abnehmenden Grenznutzen gebremst. Auch Science-Fiction-Autoren müssen sich die einfache aber ernüchternde Tatsache gefallen lassen, dass die aufzuwendende Energie, um ein Raumschiff auf Lichtgeschwindigkeit $\mathrm{zu}$ beschleunigen, unendlich groß sein müsste.

In medizinischer Terminologie: Die kurative Resektion eines Tumors kostet 10.000 EUR und bedingt $80 \%$ der Varianz des Langzeitüberlebens, die adjuvante Chemotherapie kostet 20.000 EUR und bedingt weitere $5 \%$, eine neue Immuntherapie kostet 40.000 EUR, bedingt jedoch nur noch $1 \%$. Bei vielen Erkrankungen und Verletzungen kann die Restvarianz mit dem verfügbaren Wissen nur unbefriedigend erklärt werden - die biologische Variabilität (wie z. B. die genetische Ausstattung) stellt hierbei sicher eine wesentliche Komponente dar.

Auch durch Aufwendung maximaler Mittel kann aber kein Zustand oberhalb von Gesundheit erreicht werden - es ist undenkbar, gesünder als gesund zu sein (oder, um bei der Definition der Weltgesundheitsorganisation zu bleiben, weniger als keine gesundheitliche Einschränkung aufzuweisen).

Ebenso wie ein gesellschaftlicher Konsens über den Austausch von zwei gleichen (bzw. nicht unterlegenen, dabei innerhalb festgelegter Grenzen auch unterschiedlichen) Behandlungsmethoden erforderlich ist, sollten alle Partner im Gesundheitswesen darüber entscheiden, um wie viel besser eine neue Methode sein muss, um die zu erwartenden Mehrausgaben zu akzeptieren.

\section{Ebenen der Gesundheitstechnologiebewertung}

Es ist hilfreich, in die Diskussion um Qualität und Nutzen die unterschiedlichen Ebenen der Gesundheitstechnologiebewertung („efficacy“, „effectiveness“, „,efficiency“) einfließen zu lassen (• Tab. 1). Nur eine Methode, die auch jenseits der kontrollierten Studiensituation - also unter Alltags- und Populationsbedingungen - noch einen Nutzwert besitzt, wird auch

Trauma Berufskrankh 2008 · 10[Suppl 1]:85-90 DOI 10.1007/s10039-007-1305-5

(c) Springer Medizin Verlag 2007

\section{A. Ekkernkamp · E. Haider - E. Froese - D. Stengel Qualität und Nutzen der Heilverfahren}

\section{Zusammenfassung}

Das bundesdeutsche Gesundheitssystem nimmt im internationalen Vergleich - mit einer flächendeckenden Versorgung auf allen Stufen unabhängig von Sozialstatus und Herkunft - eine Spitzenstellung ein. Der medizinische Sektor (die Gesundheitswirtschaft) sowie die akademische und industrielle Forschung und Entwicklung stellen einen bedeutenden Wirtschaftszweig dar. Globalisierung, wirtschaftliche Zwänge und harte medizinische Fakten erfordern Anpassungen auf verschiedenen Ebenen, um auch in Zukunft eine Versorgung auf dem neuesten Stand der Wissenschaft und unter Wahrung des Solidarprinzips gewährleisten zu können. Der Qualitätsgedanke und die Abwägung des Nutzens,

\section{Quality and benefit of treatments}

\section{Abstract}

The German healthcare service ranks high in international comparison, with thoroughgoing care supplied to patients at all stages regardless of social status and origin. The medical sector (health economics) and academic and industrial research and development together make up a significant branch of the economy. Globalisation, economic forces and hard medical facts demand adjustments at various levels if we want to be able to guarantee care that is scientifically up to date while retaining our principle of solidarity. The thought of quality and the weighing of benefit, of new medical methods, for exam- z. B. neuer medizinischer Methoden, spielen dabei eine große Rolle. Das berufsgenossenschaftliche Heilverfahren auf der Grundlage des SGB VII nimmt innerhalb des Gesundheitssystems eine besondere Stellung ein. Eine Systemevaluation und der daraus resultierende Einsatz gesichert wirksamer Komponenten können dazu beitragen, die Effektivität, Flexibilität und Glaubwürdigkeit der Heilverfahren zu erhöhen.

\section{Schlüsselwörter}

Gesundheitssystem · Berufsgenossenschaftliche Heilverfahren · Qualität · Nutzen · Systembewertungen ple, have an important role in this. The treatments approved by the employers' liability insurance associations as laid down in SGB VII have a particular position within the healthcare system. System evaluation and the consequent use of components found to be definitely effective can contribute to an enhancement of the effectiveness, flexibility and credibility of the treatments.

\section{Keywords}

Healthcare system · Treatments approved by employers' liability insurance associations . Quality · Benefit · System evaluations 


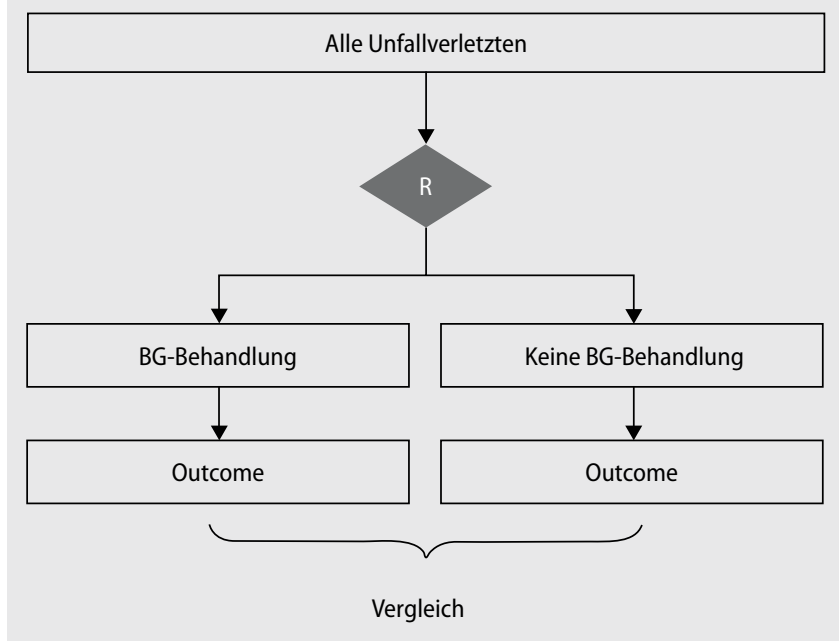

Abb. 14 Klassische randomisierte Studie. In diesem Design würden alle Verletzten, unabhängig von ihrem Versicherungsstatus, zufällig einem der beiden Heilverfahren zugeordnet werden. Obwohl dieses Design die kausale Bedeutung der BG-Behandlung für die Ergebnisqualität nachweisen würde, ist die Umsetzung aufgrund gesetzlicher Vorgaben unmöglich
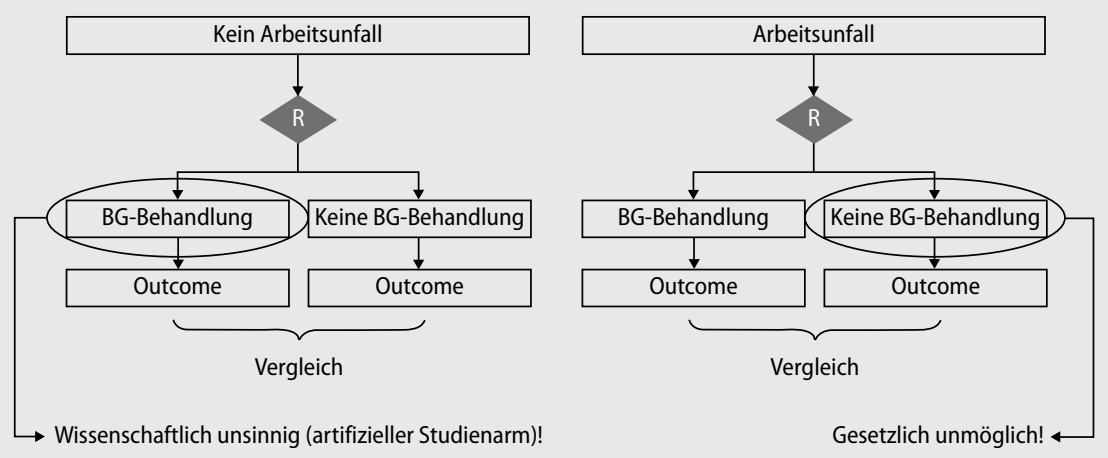

Abb. $2 \Delta$ Alternative Designs. Denkbar wäre die zufällige Zuteilung von Nicht-BG-Versicherten zu einem Standard- und einem „, add-on"-BG-Arm. Auch könnten BG-Versicherte einem „drop-off"-BGArm zugeordnet werden. Beide Designs sind weder gesetzlich möglich noch wissenschaftlich sinnvoll, da artifizielle Gruppen generiert werden

Einfluss auf die Versorgungsqualität nehmen können.

\section{Systembewertungen}

Ebenso wie in anderen Ländern (Beispiel USA: Medicare und Medicaid, Health Maintenance Organisations, Veterans' Affairs) tragen zum bundesdeutschen Gesundheitssystem unterschiedliche Leistungsträger mit verschiedenen Leistungsspektren bei. Weltweit sicher einzigartig sind die scharf umrissenen Zielvorgaben der Unfallbehandlung und -rehabilitation in Folge gesetzlich unfallversicherter Tätigkeiten [3].

Dem Arbeitsunfall kommt in Deutschland eine Sonderstellung zu. In $\$ 1$ Siebtes Buch Sozialgesetzbuch (SGB VII) heißt es:

Aufgabe der Unfallversicherung ist es, nach Maßgabe der Vorschriften dieses Buches... nach Eintritt von Arbeitsunfäl- len oder Berufskrankheiten die Gesundheit und die Leistungsfähigkeit der Versicherten mit allen geeigneten Mitteln wiederherzustellen und sie oder ihre Hinterbliebenen durch Geldleistungen zu entschädigen.

In $\$ 34$ Abs. 1 S. 1 und 2 wird ferner gesetzlich festgelegt:

Die Unfallversicherungsträger haben alle Maßnahmen zu treffen, durch die eine möglichst frühzeitig nach dem Versicherungsfall einsetzende und sachgemäße Heilbehandlung und, soweit erforderlich, besondere unfallmedizinische... Behandlung gewährleistet wird. Sie können zu diesem Zweck die von den Ärzten und Krankenhäusern zu erfüllenden Voraussetzungen im Hinblick auf die fachliche Befähigung, die sächliche und personelle Ausstattung sowie die zu übernehmenden Pflichten festlegen.

Bereits aus diesen Grundprinzipien des SGB VII lässt sich ein klarer Qualitätsge- danke ableiten - es müssen alle Strukturen und Prozesse geschaffen werden, um die Ergebnisqualität zu optimieren.

Nur für wenige Prinzipien der sozialen Sicherung besteht ein derart breiter gesellschaftlicher Konsens wie für den beschriebenen Grundsatz, durch kurzfristige finanzielle, personelle und logistische Mehrinvestition langfristig einen nachweisbaren und nachhaltigen Nutzen zu produzieren.

Der Arbeitende erbringt eine Leistung im Interesse und innerhalb des organisatorischen Rahmens eines Unternehmens. Erleidet er während dieser Leistungserbringung eine behandlungsbedürftige Verletzung, tritt die gesetzliche Unfallversicherung an die Stelle der unternehmerischen Haftungspflicht und kommt für die Behandlungskosten auf. Falls auch durch die derzeit bestmögliche Behandlung keine Wiederherstellung erzielt werden kann, muss im Rahmen der Möglichkeiten über eine Entschädigung nachgedacht werden. Das Ziel der Heilbehandlung ergibt sich von selbst: den Verletzten dorthin zurückzuführen, wo er seine Verletzung erlitten hat. Hierbei dominiert der Arbeitsplatz für den gesellschaftlichen Fortbestand weit über alle anderen denkbaren Dimensionen. Arbeitsfähigkeit ist zugleich einer der stärksten Indikatoren für Autonomie, Selbstbestimmung und Selbstbewusstsein und damit nicht nur aus wirtschaftlicher, sondern auch sozialer und psychologischer Sicht vorrangig anzustreben.

Im Interesse der Erreichung dieser Ziele stellen die Steuerung und Lenkung der Heilbehandlung und der Rehabilitation und damit auch der rationale Einsatz von Mitteln seit jeher ein zentrales, in der Praxis etabliertes Element der gesetzlichen Unfallversicherung dar.

Ein Nachweis des Nutzens der spezifischen Komponenten und des hiermit verbundenen Aufwands des sog. berufsgenossenschaftlichen Heilverfahrens lässt sich jedoch nur mit belastbaren Daten führen, die auch höchsten Ansprüchen an die Gesundheitstechnologiebewertung genügen. Es entspricht dem Selbstverständnis der gesetzlichen Unfallversicherung, sich dieser Herausforderung zu stellen und die geforderten Daten zu generieren. 
Abb. $3 \rightarrow$ Mögliches Studiendesign für einen direkten Systemvergleich. Es werden

Nicht-BG- und BG-Versicherte mit gleichem Verletzungsmuster identifiziert. Nach Adjustierung für biologische Ungleichgewichte lässt sich der Effekt der BGBehandlung erkennen

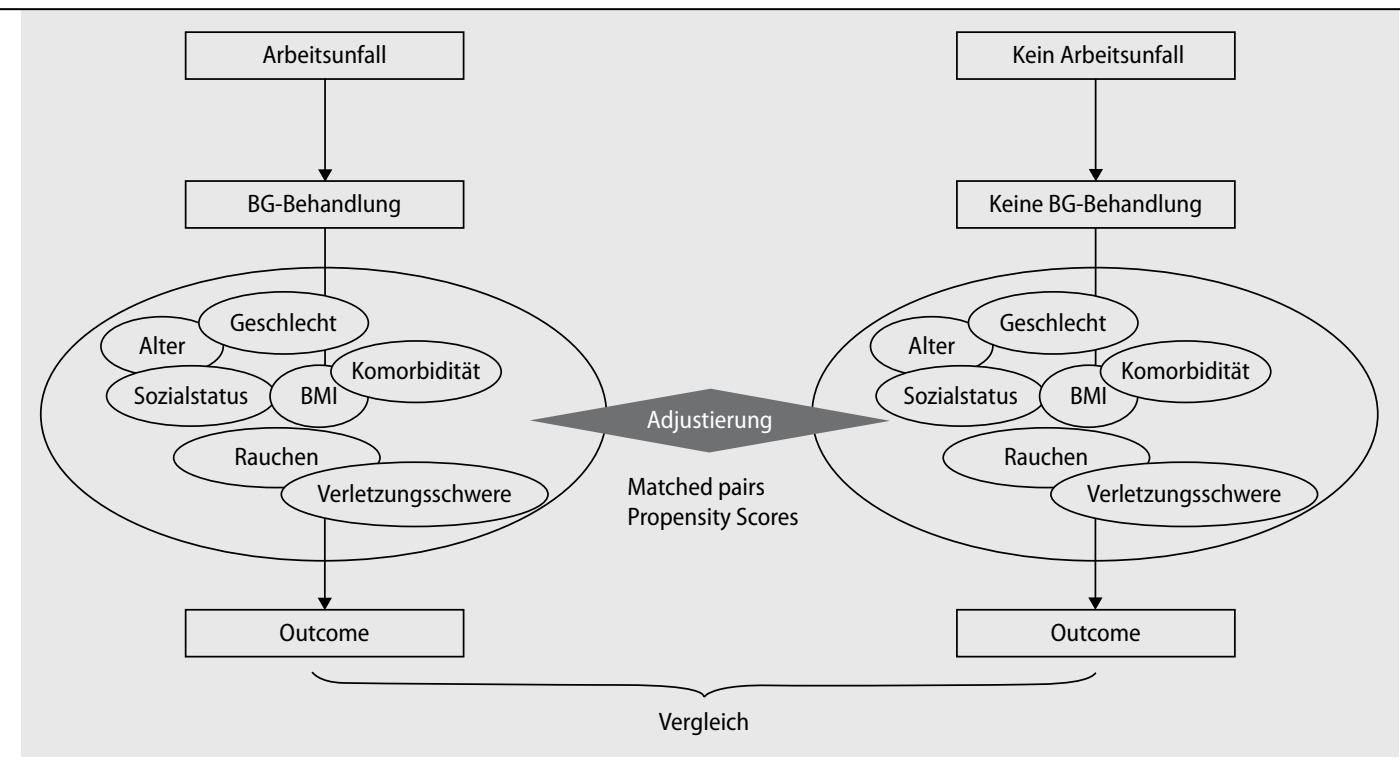

Ziel eines epidemiologisch unangreifbaren Studienaufbaus ist der Nachweis, dass nach dem Eintritt einer Unfallverletzung durch die spezifischen Komponenten des berufsgenossenschaftlichen Heilverfahrens auf der Grundlage des SGB VII die konditionale Wahrscheinlichkeit (,gegeben, dass..."), die geforderten Ziele zu erreichen, höher ist als bei Heilverfahren außerhalb des SGB VII.

\section{Vergleich der Heilverfahren innerhalb und außerhalb des SGB VII}

Eine wissenschaftlich fundierte, politisch tragfähige und machbare Annäherung an das Problem könnte darin bestehen, die Behandlungsergebnisse bei ausgewählten Verletzungen zwischen Heilverfahren innerhalb und Heilverfahren außerhalb des SGB VII zu vergleichen. Dies ist deshalb möglich (und auch sinnvoll), weil jede Verletzung am Arbeitsplatz natürlich auch unter allen anderen Lebensbedingungen eintreten kann. Es sollte sich dabei um Verletzungen handeln, bei denen aufgrund des unterschiedlichen Managements nach der Krankenhausbehandlung Kontraste zwischen den Heilverfahren zu erwarten sind.

Werden Betroffene mit schweren Verletzungen (z. B. Polytrauma, Rückenmarkverletzungen, offenen und gelenknahen Frakturen, Verbrennungen) am berufsgenossenschaftlichen Zentrum behandelt, profitieren sie mit hoher Wahr- scheinlichkeit vom dortigen Wissen, Können und der apparativen Ausstattung [4]. Unterschiede werden sich daher erst bei der Nachverfolgung der Betroffenen erkennen lassen, wenn sich die Wege zwischen der Heilbehandlung nach SGB VII und der Heilbehandlung nach anderen Rechtsgrundlagen trennen.

Stellt man die Gleichverteilung biologischer Risikofaktoren (Alter, Geschlecht, Body-Mass-Index, Komorbidität usw.) sicher, erklären das individuelle Heilverfahren bzw. seine Komponenten die beobachteten Unterschiede in der Ergebnisqualität. Die Behandlung außerhalb des Regelungsrahmens des SGB VII wird hierbei als Referenzstandard, die Behandlung auf der Grundlage des SGB VII als experimentelle Intervention betrachtet.

\section{Studiendesigns}

In der klinischen Forschung wird diese Gleichverteilung im Idealfall durch eine zufällige Zuteilung geeigneter Probanden zu den zu prüfenden Verfahren (Randomisierung) erreicht. Die bestechende Idee der randomisierten Studie (oder dem klinischen Experiment) liegt darin, dass neben den bekannten auch alle unbekannten Störvariablen gleichmäßig auf beide Therapiegruppen verteilt werden. Dies garantiert, dass beobachtete Unterschiede auf einen Therapieeffekt, nicht auf ein Ungleichgewicht in derzeit nicht messbaren Variablen, zurückgeführt werden können.
Aufgrund der gesetzlichen Vorgaben kann ein Systemvergleich jedoch nicht primär über eine randomisierte Studie realisiert werden. Die $\mathbf{D}$ Abb. 1 und 2 stellen die wissenschaftlich wünschenswerten, aber praktisch nicht umsetzbaren Studienmodelle dar.

Die randomisierte Studie kann jedoch verwendet werden, um Dosis-WirkungsBeziehungen bestimmter Komponenten des berufsgenossenschaftlichen Heilverfahrens darzustellen und somit indirekt einen kausalen Zusammenhang zwischen Prozess- und Ergebnisqualität abzubilden. Beispiele für diese Komponenten sind das Reha-Management, die erweiterte ambulante Physiotherapie, die berufsgenossenschaftliche stationäre Weiterbehandlung oder die Evaluation der funktionellen Leistungsfähigkeit (EFL). Denkbar wäre der Vergleich zwischen einem Standardtherapiearm mit allen etablierten Komponenten des BG-Verfahrens und einem "Hochdosis"-Therapiearm, in dem besonders wirksame Prozesse noch häufiger und zeitintensiver umgesetzt werden.

Der direkte Vergleich der Ergebnisqualität muss mit anderen Mitteln erbracht werden. Es existieren Methoden (wie z. B. Regressionsverfahren oder „propensity score"), um das Profil von zwei Kohorten so anzugleichen, dass die interessierende Intervention zumindest einen Großteil der beobachteten Unterschiede erklärt (- Abb. 3).

An entsprechenden Institutionen könnten also Betroffene mit gleichen Ver- 
letzungen identifiziert werden, die unter den Bedingungen von SGB VII oder nach anderen Grundlagen behandelt wurden. Es ist sinnvoll, eine derartige Untersuchung retrospektiv zu führen, da so bereits auf verfügbare administrative Daten zurückgegriffen werden kann. Die Dauer der Studie kann zudem erheblich reduziert werden, da das Nachbeobachtungsintervall bis zur Erreichung von Endpunkten bereits überschritten wurde.

Die geeigneten Endpunkte müssen sowohl auf die spezifischen Ziele der Heilverfahren (d. h., Dauer der Arbeitsunfähigkeit bzw. Eintritt der Arbeitsfähigkeit, Berentung) zugeschnitten sein, als auch akzeptierte Instrumente zur Messung der Funktion [z. B. „Disabilities of the Arm, Shoulder and Hand" (DASH)-Fragebogen], der gesundheitsbezogenen Lebensqualität (z. B. Short Form 36 oder EuroQol) und Kosten-Nutzen-Verhältnisse [z. B. "quality-adjusted life years" (QALY)] umfassen.

\section{Fazit}

Qualität in der Medizin ist untrennbar mit Nutzen - und damit auch dem wissenschaftlichen Nachweis von Nutzen - verbunden. Eine Systemevaluation ist dringend erforderlich, um diejenigen Strukturen und Prozesse zu identifizieren, die den größten Teil der Varianz in der Ergebnisqualität des berufsgenossenschaftlichen Heilverfahrens erklären. Ein gezielter Einsatz dieser gesichert wirksamen Komponenten könnte, unabhängig von gesetzlichen Vorgaben, dessen Effektivität, Flexibilität und Glaubwürdigkeit auch im internationalen Vergleich erhöhen.

\section{Korrespondenzadresse}

\section{Prof. Dr. E. Haider}

Zentrum für Klinische Forschung,

Unfallkrankenhaus Berlin,

Warener Straße 7, 12683 Berlin

haider@ukb.de

Interessenkonflikt. Der korrespondierende Autor gibt an, dass kein Interessenkonflikt besteht.

\section{Literatur}

1. Contopoulos-loannidis DG, Ntzani E, loannidis JP (2003) Translation of highly promising basic science research into clinical applications. Am J Med 114: 477-484

2. Derrett S, Paul C, Morris JM (1999) Waiting for elective surgery: effects on health-related quality of life. Int J Qual Health Care 11: 47-57

3. Ekkernkamp A (2007) Aufgaben der Traumatologie aus Sicht des SGB V und SGB VII. Die BG 10: 225226

4. Haider E, Froese E (2007) Auftrag und Leistungsspektrum der BG-Kliniken. Die BG 10: 218-219

5. Lofvendahl S, Eckerlund I, Hansagi H et al. (2005) Waiting for orthopaedic surgery: factors associated with waiting times and patients' opinion. Int J Qual Health Care 17: 133-140

6. Oudhoff JP, Timmermans DR, Bijnen AB, van der Wal $G$ (2004) Waiting for elective general surgery: physical, psychological and social consequences. ANZ J Surg 74: 361-367

7. Oudhoff JP, Timmermans DR, Knol DL (2007) Prioritising patients on surgical waiting lists: a conjoint analysis study on the priority judgements of patients, surgeons, occupational physicians, and general practitioners. Soc Sci Med 64: 1863-1875

8. Oudhoff JP, Timmermans DR, Knol DL et al. (2007) Waiting for elective general surgery: impact on health related quality of life and psychosocial consequences. BMC Public Health 7: 164

9. Oudhoff JP, Timmermans DR, Rietberg $M$ et al. (2007) The acceptability of waiting times for elective general surgery and the appropriateness of prioritising patients. BMC Health Serv Res 7: 32

10. Snider MG, MacDonald SJ, Pototschnik R (2005) Waiting times and patient perspectives for total hip and knee arthroplasty in rural and urban Ontario. Can J Surg 48: 355-360 